\title{
钯催化的脂肪醇的芳基化反应研究进展
}

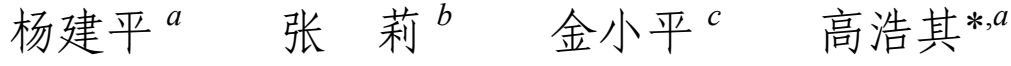 \\ 房江华 $a$ 李瑞丰 $b$ 方烨汶*,a,d \\ ( ${ }^{a}$ 宁波工程学院化学工程学院 宁波 315016) \\ $\left({ }^{b}\right.$ 太原理工大学化学化工学院 太原 030024) \\ ( 浙江医药高等专科学校基础部 宁波 315100)

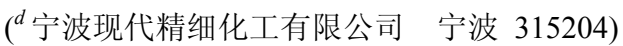

\begin{abstract}
摘要 烷基芳基醚结构广泛存在于天然和人工药物中, Pd 催化的脂肪醇的芳基化反应是合成这类化合物的重要方法之 一. 重点综述了成功运用于脂肪醇的 $\mathrm{C}-\mathrm{O}$ 偶联反应的四大类配体, 对 $\mathrm{C}-\mathrm{O}$ 偶联反应的应用进行了概述, 对该类反应 研究前景也进行了展望.
\end{abstract}

关键词＼cjkstart钯催化; 烷基芳基醚; $\mathrm{C}-\mathrm{O}$ 偶联反应; 膦配体

\section{Recent Developments on Pd-Catalyzed Arylations of Aliphatic Alcohols}

\author{
Yang, Jianping ${ }^{a} \quad{\text { Zhang, } \mathrm{Li}^{b} \quad \text { Jin, Xiaoping }}^{c} \quad$ Gao, Haoqi*a \\ Fang, Jianghua $^{a} \quad$ Li, Ruifeng $^{c} \quad$ Fang, Yewen ${ }^{*, a, d}$ \\ ( ${ }^{a}$ School of Chemical Engineering, Ningbo University of Technology, Ningbo 315016) \\ ( ${ }^{b}$ College of Chemistry and Chemical Engineering, Taiyuan University of Technology, Taiyuan 030024) \\ ( ${ }^{c}$ Department of Basic Education, Zhejiang Pharmaceutical College, Ningbo 315100) \\ $\left({ }^{d}\right.$ Ningbo Current Tech Fine Chemical Co. Ltd., Ningbo 315204)
}

\begin{abstract}
Aromatic ethers of aliphatic alcohols represent an important structural motif in many naturally occurring and medicinally relevant compounds. Palladium-catalyzed aliphatic alcohol arylation is a kind of important methods to access them. This review focuses on four types of phosphorus ligands successfully employed in $\mathrm{C}-\mathrm{O}$ cross coupling reactions of aliphatic alcohols. The applications of $\mathrm{C}-\mathrm{O}$ cross coupling reactions are summarized and the reaction potential research areas are also discussed.
\end{abstract}

Keywords palladium catalysis; alkyl aryl ether; $\mathrm{C}-\mathrm{O}$ cross-coupling; phosphorus ligand

烷基芳基醚(Chart 1)是有机合成中一类常见且重要 的结构片段，它广泛存在于天然和人工合成药物中 ${ }^{[1]}$. 因此，在过去一个多世纪里围绕烷基芳基醚的合成产生 了大量的方法. 常用的方法有 Williamson 醚合成法 ${ }^{[2 a]}$ 、 传统 Ullmann 偶联法 ${ }^{[2 b]}$ 、Chan-Lam 氧化偶联反应 ${ }^{[2 c]}$ 、 芳基亲核取代反应 ${ }^{[2 \mathrm{~d}]} 、$ Mitsunobu 反应 ${ }^{[2 \mathrm{e}]}$ 、磺酰基转移反 应 ${ }^{[2]}$ 等 $^{[2 \mathrm{~g}, 2 \mathrm{~h}, 2 \mathrm{i}]}$. 上述反应都是化学计量反应, 在反应的
经济性、普适性和官能团兼容性等方面存在较多不足. 正是由于这些方法在合成烷基芳基醚上的缺陷，催化型 的合成方法在过去近 20 年中得到了研究界的重视并取 得了较大的突破 ${ }^{[1]}$. 催化型反应的研究策略主要有两种: （1） C(Ar)-X 键的烷氧基化，即过渡金属催化的芳基卤 代物和脂肪醇的偶联反应; (2) $\mathrm{C}(\mathrm{Ar})-\mathrm{H}$ 键的烷氧基化, 即过渡金属催化的芳烃碳氢键的官能团化反应.由于运

\footnotetext{
*E-mail: nbut.fang@gmail.com

Received January 7, 2013; revised February 25, 2013; published online March 8, 2013.

Project supported by the National Natural Science Foundation of China (No. 21202090), the Science and Technology Innovation Team of Ningbo City (No. 2011B82002), the Natural Science Foundation of Zhejiang Province (No. LY12B02001), and the Natural Science Foundation of Ningbo City (Nos. 2011A610123, 2012A610123).

国家自然科学基金(No. 21202090)、宁波市科技创新团队(No. 2011B82002)、浙江省自然科学基金(No. LY12B02001)、宁波市自然科学基金(Nos.
} 2011A610123, 2012A610123)资助项目. 
用 $\mathrm{C}-\mathrm{H}$ 键活化策略合成烷基芳基醚只有零星的报道, 研究并不系统, 而且多数运用了邻位诱导策略, 因此在 这里不做讨论 ${ }^{[3]}$. 尽管 $\mathrm{Cu}$ 催化的 $\mathrm{C}-\mathrm{O}$ 反应取得了长足 的进展, 但从反应活性角度来看, 特别是叔醇等大位阻 醇作为亲核体参与的偶联反应以及氯代烯烃和芳烃等 反应活性较低的亲电体参与的偶联反应, Ullmann 反应 催化体系常常遇到反应效率低等问题 ${ }^{[4]}$. 因此, 本综述 重点讨论 Pd 催化的芳基卤代物和脂肪醇的 $\mathrm{C}-\mathrm{O}$ 偶联 反应.<smiles>COc1ccc2cc(CCC(C)=O)ccc2c1</smiles><smiles>Cc1cc(O[Na])c(CC(N)[18OH])cc1O[Na]</smiles>

2,5-di([D $\left.\mathrm{D}_{3}\right]$ methoxy)-4methylamphetamine<smiles>CC(C)(C)Oc1ccc(Cl)cc1C(=O)NCc1ccc(S(=O)(=O)NC(=O)NC2CCC(O)CC2)cc1</smiles>

Chart 1

与 $\mathrm{C}-\mathrm{C}, \mathrm{C}-\mathrm{N}$ 等偶联反应一样, 配体的选择是 $\mathrm{C}-\mathrm{O}$ 偶联反应成败的关键, 根据已经报道的成功例子, 目前用于 $\mathrm{C}-\mathrm{O}$ 偶联反应的配体主要集中在大位阻且富 电子的单膦和双膦等配体, 比较成功的有 Buchwald 小 组基于联苯骨架的单膦配体、Singer 和 Beller 两个小组 的二吡唑类单膦配体、Hartwig 小组的二茂铁类单膦配 体、Merck 公司研究小组应用的 Josiphos 双膦配体等, 本 综述将主要围绕这几个小组的工作, 着眼于配体的设计 与选择、机理研究和在有机合成中的应用三方面进行概 述、比较和讨论.

\section{1 膦配体的发展}

\subsection{Buchwald 催化体系}

作为最早参与 $\mathrm{Pd}$ 催化 $\mathrm{C}-\mathrm{O}$ 偶联的小组之一, 1996 年 Buchwald 小组和 Hartwig 小组分别报道了脂肪醇及 其盐的芳基化反应 ${ }^{[5]}$. 在 Buchwald 小组 ${ }^{[5]}$ 的工作中, 他 们发现双膦配体 Tol-BINAP (L1)和 DPPF (L2) 可以有 效的促进 $\mathrm{C}-\mathrm{O}$ 偶联反应的发生(Eq. 1). 特别是叔醇和 芳基溴的分子内偶联反应, 反应效率最高. 即使是七元
氧环的底物，在相同的催化体系下，也以中等收率得到 七元环的芳基醚. 从下列对照反应可以看出，从反应效 率上讲, 仲醇比叔醇要低的多. 这主要是由于以仲醇为 底物时, $\beta-\mathrm{H}$ 消除反应作为一个环合反应的主要竞争性 副反应，消耗了一部分原料，从而导致目标偶联反应效 率降低.

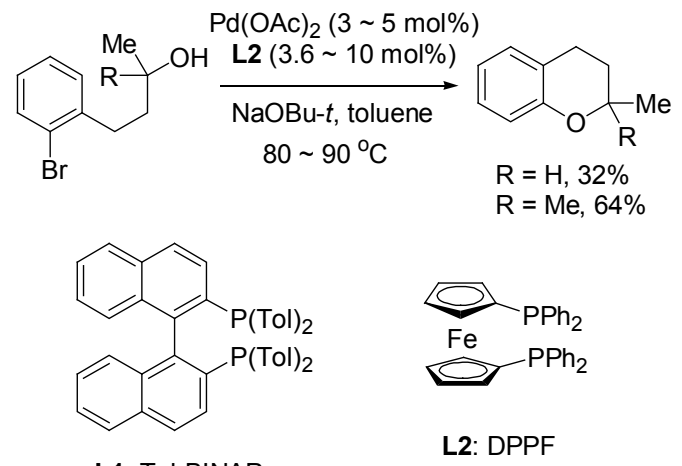

当对叔醇类底物进行环合反应研究时, Tol-BINAP 和 DPPF 可以有效地促进分子内 $\mathrm{C}-\mathrm{O}$ 偶联反应的发生, 但对于更富挑战性的伯醇和仲醇类底物, 却难以有效地 抑制 $\beta-\mathrm{H}$ 消除副反应的发生. Buchwald 小组 ${ }^{[6]}$ 受他们 $\mathrm{C}-$ $\mathrm{N}$ 偶联反应的启发，研究发现：基于联苯和联荎骨架的 二叔丁基取代的单膦配体可以有效地抑制 $\beta-\mathrm{H}$ 消除副反 应的发生，使反应朝环合偶联方向进行(Scheme 1). 例如 当以 2-溴苯乙醇为底物时, 在用 Tol-BINAP 或 DPPF 为 配体时, 主要进行的是 $\beta-\mathrm{H}$ 消除反应; 而当以 $\mathbf{L 3}$ 为配体 时，可以高效地得到目标偶联产物(Scheme 1).

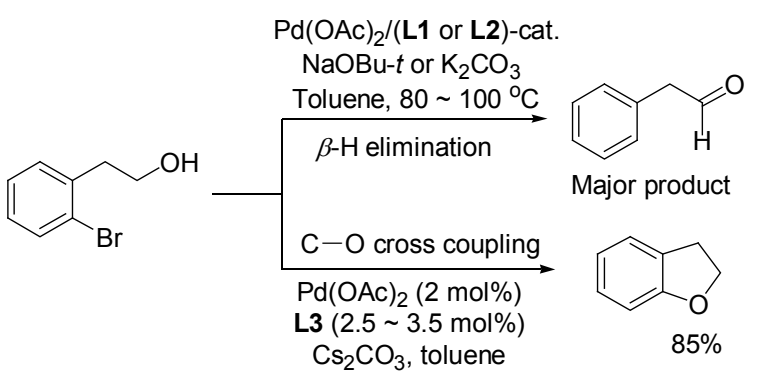

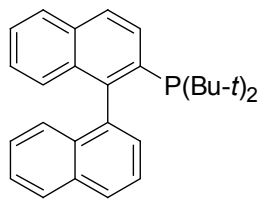

L3

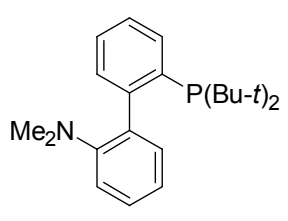

L5

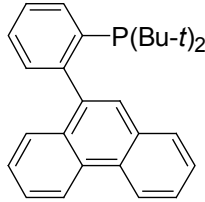

L4

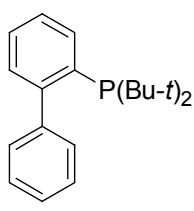

Scheme 1 
当以光学纯的醇进行偶联环合时, 由于 $\beta-\mathrm{H}$ 消除反 应及其可逆性特征, 反应往往伴随着消旋化(Eq. 2$)^{[7]}$. 因此, 如何有效地抑制 $\beta-\mathrm{H}$ 消除反应, 对拓宽 $\mathrm{C}-\mathrm{O}$ 偶 联反应在有机合成中的应用就显得格外重要. 需要指出 的是, 除配体的影响外, 偶联反应的其它众多要素, 如 Pd 催化剂前体、碱、反应温度对反应的消旋化都有一定 影响(Scheme 2). 因此, $\beta$-H 消除反应是各个偶联反应要 素单独或共同作用的结果.
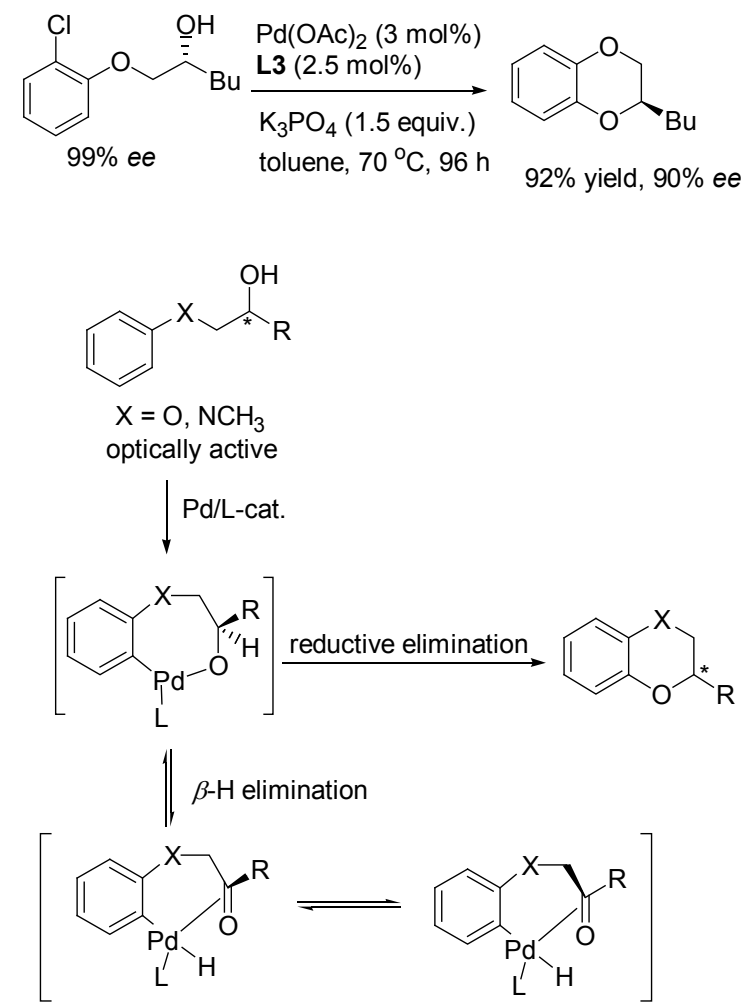

Scheme 2

在先前的工作基础上, Buchwald 小组 ${ }^{[8]}$ 于 2005 年发 展了基于联苯和 $\alpha$-芳基萗为骨架的单膦配体(Chart 2), 由此进一步拓展了脂肪醇的 $\mathrm{C}-\mathrm{O}$ 偶联反应. 相比于 2000 年的配体, 无论在底物适用范围, 还是反应活性方 面都有了明显的提高. 值得指出的是, 在他们的工作中, 首次实现了烯丙基醇的芳基化反应(Eq. 3). 以往运用 Pd 和 $\mathrm{Ru}$ 催化的苯酚类化合物和烯丙基化合物间的亲核取 代反应合成芳基烯丙基醚时往往会遭遇化学选择性问 题. 而以偶联反应为合成策略时, 由于该类反应的原位 反应特性, 可避免化学选择性问题, 从而有效地丰富了 合成方法学. 但不足的是, 在多数例子中, 需要用到正 三丁胺作为溶剂以抑制脱卤反应的发生. 同时, 配体的 普适性较差, 需要遵循配体一底物匹配性规则(Chart 2). 正是这些不足, 促使着他们进一步探寻和发展更高效且 更具普适性的催化体系.

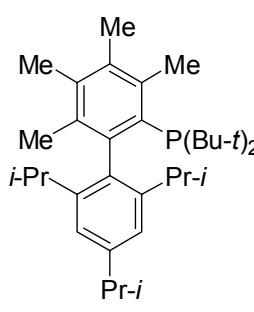

L7

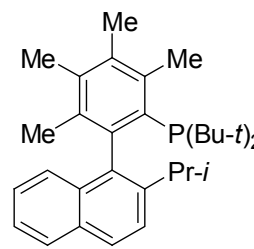

L8<smiles>Cc1c(C)c(C)c(-c2c(CBr)ccc3ccccc23)c(CCBr)c1C</smiles>

L9

\section{Chart 2}
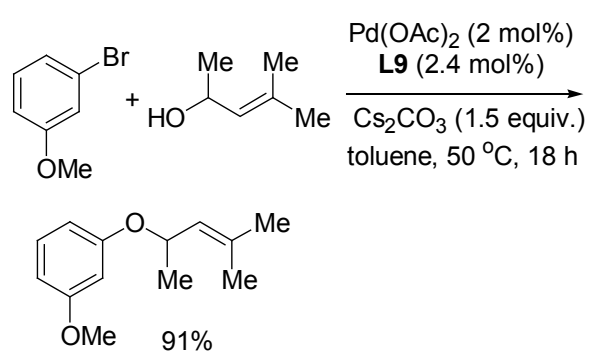

最近, Buchwald 小组 ${ }^{[9]}$ 发展了一类新型单膦配体 $t$-BuBrettPhos, 研究发现该类配体可以有效地促进还原 消除反应，从而实现 $\mathrm{C}-\mathrm{F}, \mathrm{C}-\mathrm{Br}$ 和 $\mathrm{C}-\mathrm{O}$ 等充满挑战 的偶联反应. 在这些研究工作的基础上, 他们意识到配 体 $\mathrm{Me}_{4}-(t-\mathrm{Bu}) \mathrm{XPhos}$ 中含膦的芳环 6 位上取代基和含三 异丙基的芳环相互作用，可以使催化剂中间体的构象更 具刚性, 从而加速还原消除反应; 此外, 配体 $t$-BuBrettPhos 中膦取代的芳环 3 位上甲氧基的空间位阻 效应和对 $\mathrm{Pd}(\mathrm{II})$ 的稳定作用都有利于还原消除反应的进 行. 在这些研究结果的启发下, 他们把前面两类配体的 有利点加以综合, 即在 3 位引入甲氧基, 6 位引入甲基, 设计了新型配体 RockPhos (Chart 3) ${ }^{[10]}$.<smiles>Cc1cc(P(C)c2ccccc2)c(-c2c(C)cc(C(C)C)cc2P(C)C)c(C)c1C</smiles>

L10 ( $\mathrm{Me}_{4}-t$-BuXPhos)<smiles>COc1ccc(OC)c(C(C)C)c1-c1c(P)cc(PC)cc1P(C)C</smiles>

L11 ( $t$-BuBrettPhos)

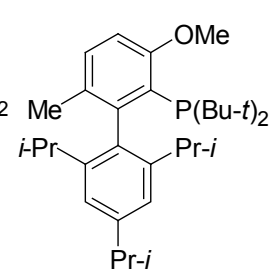

L12 (RockPhos)

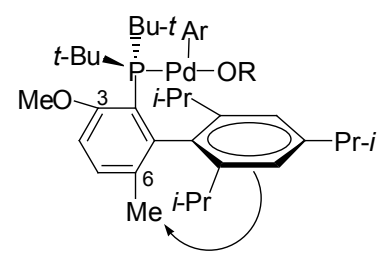

Chart 3

实验证明，即使对极富挑战性的富电子对氯苯甲醚 和 2-丁醇进行偶联反应时, 仍以较高的产率得到目标烷 
氧基芳基醚. 值得一提的是，相比于以往报道的配体 L8, 在对杂环芳基卤代物进行 $\mathrm{C}-\mathrm{O}$ 偶联比较反应时, RockPhos 配体在偶联效率上具有明显的优势(Eq. 4).

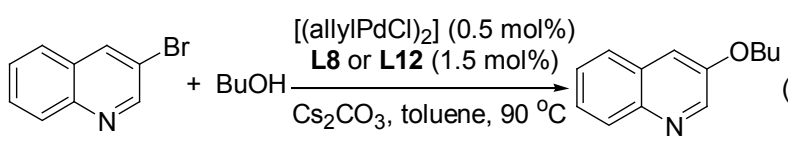

\section{L12: $98 \%$ \\ L8: $12 \%$}

最近, Peruncheralathan 小组 ${ }^{[11]}$ 将 Buchwald 小组发 展的 $t$-BuXPhos 配体成功运用于甲基芳基醚及三氛代甲 基芳基醚的合成，从而进一步拓展了 Buchwlad 单膦配 体在 $\mathrm{C}-\mathrm{O}$ 偶联反应中的应用.

\subsection{Hartwig 催化体系}

1999 年, Hartwig 小组 ${ }^{[12]}$ 考察了他们小组发展的一 类基于二茂铁为骨架的二叔丁基单膦配体 $\mathrm{FcP}(t-\mathrm{Bu})_{2}$ 在 $\mathrm{C}$ - $\mathrm{O}$ 偶联反应中的应用(Eq. 5). 研究表明, 配体不但能 有效促进芳基卤代物和酚钠的偶联反应，同时也可以用 于烷基芳基醚的合成. 有意思的是, 在 $\mathrm{Pd} / \mathrm{FcP}(t-\mathrm{Bu})_{2}$ 催 化体系下，芳基氯代物比相应的溴代物反应偶联效率要 高.

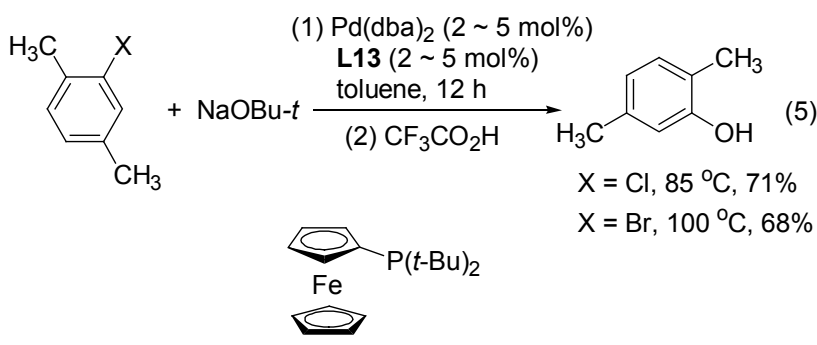

L13: $\mathrm{FcP}(t-\mathrm{Bu})_{2}$

2000 年, Hartwig 小组 ${ }^{[13]}$ 进一步对 $\mathrm{FcP}(t-\mathrm{Bu})_{2}$ 单膦配 体进行了修饰, 即在二茂铁骨架上对未取代的环戊二烯 基进行全苯基取代，得到了新型配体 $\mathrm{Ph}_{5} \mathrm{FcP}(t-\mathrm{Bu})_{2}$ (Scheme 3). 和先前的配体相比, 在催化活性、底物适用 范围和官能团兼容性方面都取得了明显突破. 例如, 运 用 $\mathrm{FcP}(t-\mathrm{Bu})_{2}$ 为配体时, 分子内 $\mathrm{C}-\mathrm{O}$ 偶联环合反应需 要在加热情况下进行; 而运用 $\mathrm{Ph}_{5} \mathrm{FcP}(t-\mathrm{Bu})_{2}$ 为配体时, 相同的反应则只要在室温下进行即可(Eq. 6). 值得一提 的是, 二茂铁类配体的合成与修饰比较容易, 这为进一 步开发更高反应活性、普适性更好的配体提供了可能.

\subsection{Singer-Beller 催化体系}

2006 年, 辉瑞公司的 Singer 研究小组在研究 C-N 偶联反应时发展了一类基于二吡唑为骨架的单膦配 体 $^{[14]}$. 2008 年, 他们进一步优化了合成路线, 从 3-苯甲 酰基苯丙酮等 1,3-二酮出发，经四步以总产率近 $60 \%$ 得
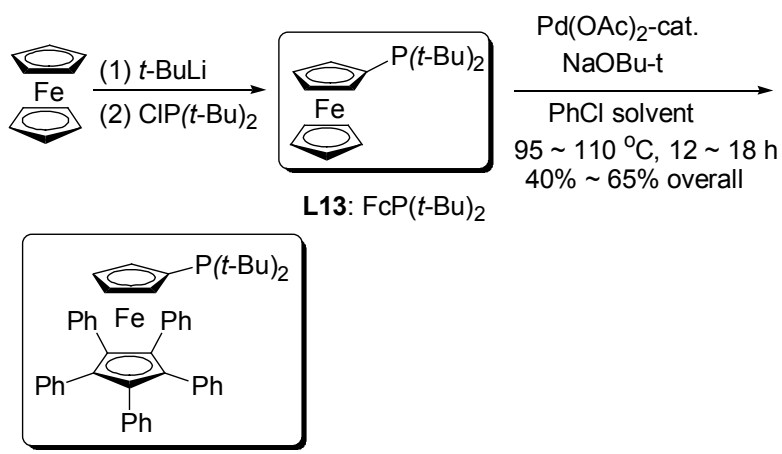

L14: $\mathrm{Ph}_{5} \mathrm{FcP}(t-\mathrm{Bu})_{2}$

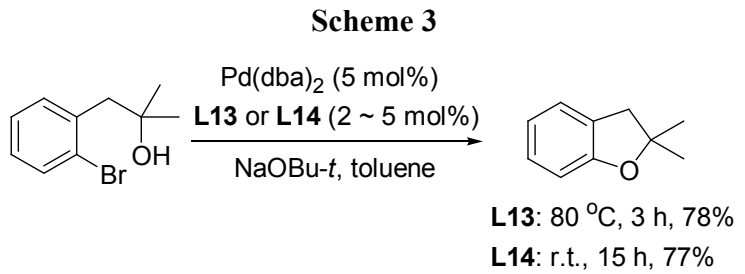

到对空气和水不敏感的以二吡唑为骨架的大位阻单膦 配体 $\mathbf{L 1 5}^{[15]}$. 该合成路线不但可以进行公斤级放大生 产，而且前三步反应只需进行一次分离提纯. 此外，该 类配体的二吡唑骨架很容易进行修饰，这些特点都极大 地提升了该类配体的应用前景. Beller 小组 ${ }^{[16]}$ 应用 Singer 小组发展的合成策略成功制备了二金刚烷基取代 的二吡唑型单膦配体 L16 (Scheme 4).

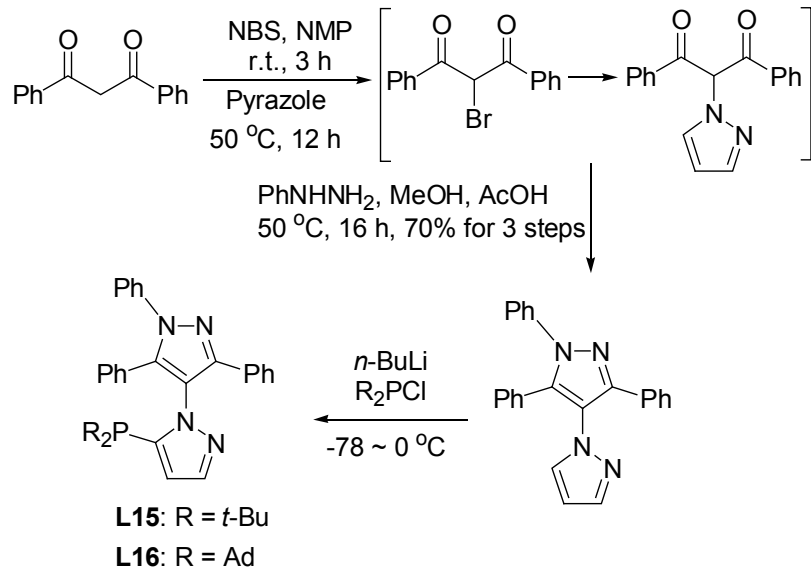

Scheme 4

在 $\mathrm{C}-\mathrm{N}, \mathrm{C}-\mathrm{C}$ 偶联反应研究基础上, Singer 小组 ${ }^{[15]}$ 考察了 Bippyphos (L15) 在 $\mathrm{C}-\mathrm{O}$ 偶联反应中的应用. 实 验表明: 缺电子的芳基卤代物可以和甲醇进行较高效偶 联; 但以富电子芳烃或大位阻芳烃为亲电偶联体时，不 但偶联效率低下，而且伴随脱卤和 $\beta$ - $\mathrm{H}$ 消除等副反 应.

2010 年, Beller 小组 ${ }^{[16]}$ 在大量基于二金刚烷基取代 的单膦、双膦配体研究工作的基础上，进一步将金刚烷 基引入了 Singer 的二吡唑单膦配体，系统考察了伯醇和 
芳基卤代烃的偶联. 从 2-溴甲苯和正丁醇的偶联反应中 可以看：相比于二叔丁基取代的 Bippyphos 配体 L15, 金刚烷基(L16)的引入极大地提升了偶联效率，可以从 中等收率提高到 $86 \%$ 的优秀产率(Eq. 7) ${ }^{[16]}$.

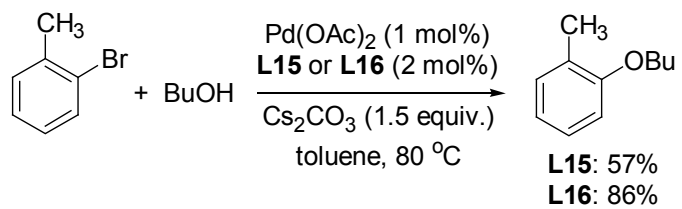

最近, Beller 小组 ${ }^{[17]}$ 用相同的催化体系实现了丙炔 醇的芳基化反应(Eq. 8). 在他们的反应条件下，位阻较 大的邻位卤代芳烃、活性较低的芳基氯代烃, 带供电或 吸电基团的卤代芳烃以及吡啶和喹啉杂环芳烃都可以 很好地作为亲电体参与偶联反应.

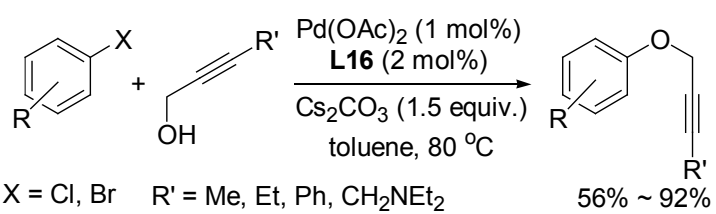

此外, Beller 小组 ${ }^{[18]}$ 进一步将他们的催化体系成功 运用于甲基芳基醚的合成. 同样, 芳基溴代或氯代物及 其芳香杂环甾代烃都能很好地和甲醇进行偶联反应. 此 外, 他们还将此催化体系运用于氞代甲醇和芳基卤代烃 的偶联, 从而进一步拓展了该催化体系在 $\mathrm{C}-\mathrm{O}$ 偶联反 应中的应用.

\subsection{Josiphos 催化体系}

在目前多数报道中, 脂肪醇的芳基化反应大多运用 的是单膦配体. 最近, Merck 公司的研究小组 ${ }^{[19]}$ 报道了 二茂铁类双膦配体 $\mathbf{L 1 7}$ 可以作为一类高效配体用于 C$\mathrm{O}$ 键偶联反应(Eq. 9). 在亲核偶联体方面, 伯、仲、叔醇 都可以进行高效偶联; 而对于亲电偶联体, 则只适用于 活化的芳基卤代烃. 当以溴苯或氯苯为偶联体时, 偶联 反应效率很低 $(<5 \%)$. 有意思的是, 通过对照反应发现: 反应的加料顺序对 Buchwald 催化体系 (RockPhos 为配 体)有较大影响, 而采用 Josiphos 为配体时, 对反应效率 影响很小.

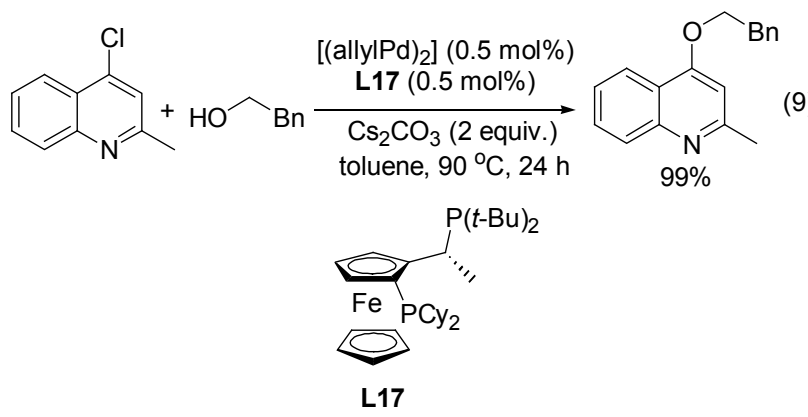

\section{5 其它单、双膦配体}

除了以上介绍的各类高效膦配体以外，其它结构简 单的膦配体如三叔丁基膦 $\left[\mathrm{P}(t-\mathrm{Bu})_{3}\right]^{[20]}$ 、三苯基膦

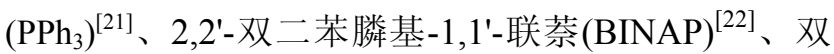
(2-二苯基膦)苯醚(DPEphos) ${ }^{[23]}$ 等也在 $\mathrm{C}-\mathrm{O}$ 偶联反应 中有成功的应用. 最近, Kündig 小组 ${ }^{[20 b]}$ 报道了利用分子 内 $\mathrm{C}-\mathrm{O}$ 偶联策略, 在钯和三叔丁基膦为配体的催化体 系下，可以高效地合成一系列 $2 H-1,4$-苯并啞嗪-3-(4H)酮. 仲醇、叔醇都能和芳基溴代物很好地进行分子内偶 联. 同时，手性醇底物在偶联环合后，没有消旋化现象 发生(Eq. 10).

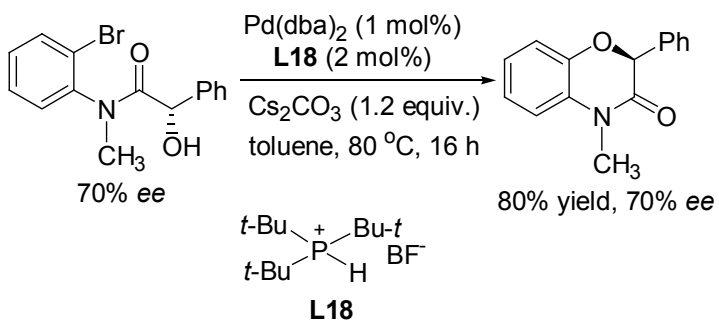

Chattopadhyay 小组 ${ }^{[22]}$ 以光学纯的天然氨基酸为起 始原料, 以 Pd-BINAP 催化的分子内 $\mathrm{C}-\mathrm{O}$ 偶联反应为 环合策略, 高效地合成了一系列氮杂七元环醚 (Eq. 11).

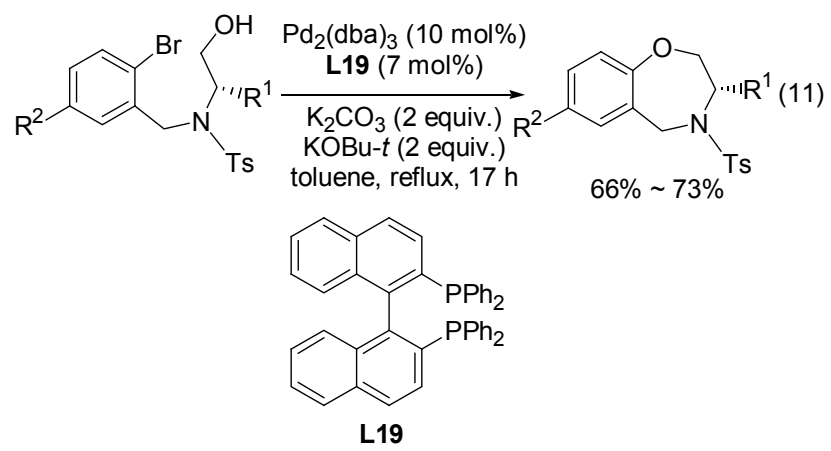

\section{C 一 O 偶联反应机理探讨}

参考 $\mathrm{C}-\mathrm{C}, \mathrm{C}-\mathrm{N}$ 等偶联反应机理, $\mathrm{C}-\mathrm{O}$ 偶联反应 也被认为是由三个基元反应组成. 由于 $\mathrm{Pd}-\mathrm{C}(\mathrm{LUMO})$ 和 $\mathrm{Pd}-\mathrm{O}(\mathrm{HOMO})$ 之间的能差较大, 所以相比于 $\mathrm{Pd}(0)$ 对芳基卤代物的氧化插入反应生成 $\mathrm{C}_{\mathrm{Ar}}-\mathrm{Pd}(\mathrm{II})-\mathrm{X}$ 和经 转金属生成 $\mathrm{C}_{\mathrm{Ar}}-\mathrm{Pd}(\mathrm{II})-\mathrm{OR}$ 这前两步反应，接下来的 还原消除生成 $\mathrm{C}-\mathrm{O}$ 偶联产物则要困难的多. 因此, 相 应的 $\mathrm{C}-\mathrm{O}$ 偶联反应机理的研究难点也主要集中在还原 消除反应这一步.

1996 年, Hartwig 小组 ${ }^{[24]}$ 推测: 芳基烷氧基 $\operatorname{Pd}(\mathrm{II})$ 络 合物在胺的芳基化催化循环里可能是重要的潜在中间 体. 考虑到 $\mathrm{Pd}$ 催化剂及相应中间体的稳定性，在选用 $\mathrm{DPPF}$ 作为配体时, $\mathrm{C}_{\mathrm{Ar}}-\mathrm{Pd}(\mathrm{II})-\mathrm{X}$ 可以很方便地合成, 
接着在 THF 中, 通过沉降 $\mathrm{NaBr}$ 后就可以得到高纯的晶 体 $\mathrm{C}_{\mathrm{Ar}}-\mathrm{Pd}(\mathrm{II})-\mathrm{OR}$. 通过芳基烷氧基 $\mathrm{Pd}(\mathrm{II})$ 络合物的合 成, 也可以看出氧化插入和转金属这两步在 Pd 催化的 $\mathrm{C}-\mathrm{O}$ 偶联反应中是比较容易实现的. 由于化合物在室 温下有很高的反应活性，因此难以分离鉴定，但通过核 磁确认即为芳基烷氧基 $\mathrm{Pd}(\mathrm{II})$ 络合物. 在室温下反应 1 $\mathrm{h}$, 即可以得到 85\%收率的叔丁基芳基醚(Scheme 5). 这 是涉及 $\mathrm{C}-\mathrm{O}$ 键形成过程中的还原消除反应的第一个直 观实例.
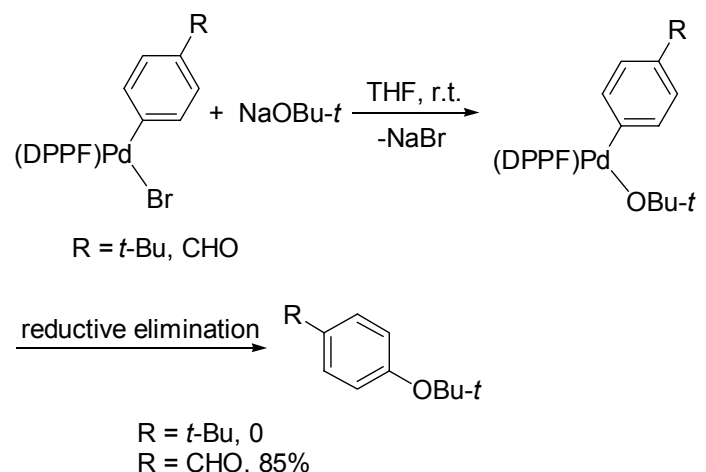

Scheme 5

几乎在差不多的时候, Buchwald 小组 ${ }^{[25]}$ 应用动力学 研究手段, 对 $\mathrm{C}-\mathrm{O}$ 偶联反应的还原消除反应进行了较 为细致的研究. 通过研究发现, 芳基烷氧基 $\mathrm{Pd}(\mathrm{II})$ 络合 物中芳基上取代基 $\left(\mathrm{R}^{\prime}\right)$ 的电性和脂肪醇 $\left(\mathrm{OCH}_{2} \mathrm{R}_{3}{ }^{\prime \prime}\right)$ 的亲 核性能对消除反应速度有明显影响，由此排除了协同反 应机理. 他们认为烷氧基负离子首先从 Pd 中心上解离, 然后亲核进攻和 Pd 相连的芳基碳，从而形成了 Meisenheimer 络合物，接着还原消除得到烷基芳基醚 (Scheme 6).

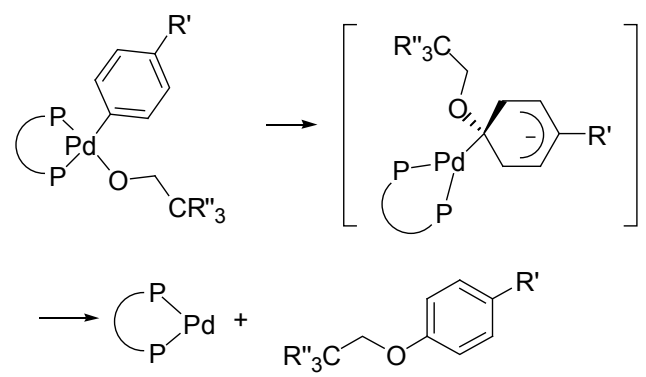

Scheme 6

在 Hartwig 和 Buchwald 两个小组开展的有关 $\mathrm{C}-\mathrm{O}$ 偶联反应机理研究的基础上, 参考其它相关偶联反应机 理，目前普遍接受的 $\mathrm{C}-\mathrm{O}$ 偶联反应机理如 Scheme 7 所 示. 从下面描述的反应机理可以看出, 还原消除反应的 快速发生，可以有效抑制 $\beta$-H 消除反应、卤代芳烃脱卤 等副反应的发生, 从而提高偶联反应效率. 在以手性醇 为偶联底物时，还可以抑制产物的消旋化.

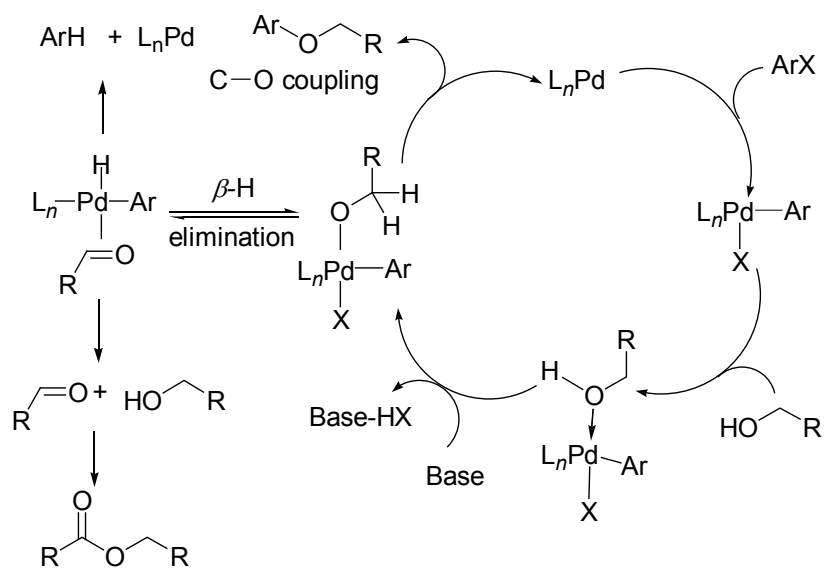

Scheme 7

\section{$3 \mathrm{C}$ - $\mathrm{O}$ 偶联反应的应用}

\section{1 多元醇的选择性偶联}

化学选择性是有机反应中的常见问题, 但在多元醇 的 $\mathrm{C}-\mathrm{O}$ 偶联反应中, Buchwald 和 Beller 小组 ${ }^{[8,16]}$ 成功地 解决了多元醇底物的化学选择性问题. Buchwald 小组运 用 $\mathrm{Pd} / \mathbf{L} 7$ 催化体系时，当醇偶联体中同时存在伯醇和仲 醇羟基时，该催化体系可以专一地促进伯醇的偶联反 应，体现了很高的化学选择性(Eq. 12). Beller 研究小组 也报道了类似的研究结果. 这对烷基多元醇无保护基状 态下进行选择性偶联具有很强的实用性.
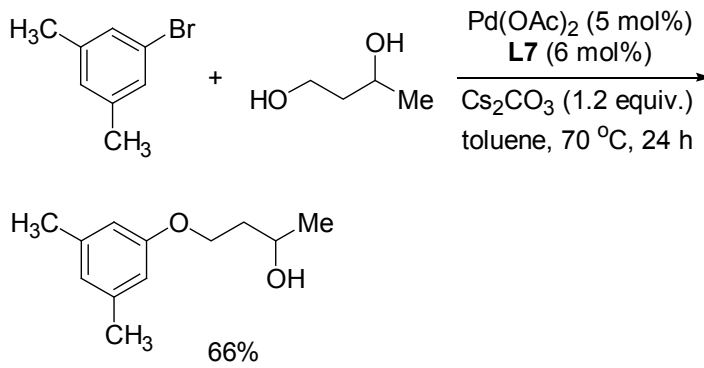

\section{2 和 UIImann 反应串联使用}

和 $\mathrm{Cu}$ 催化体系相比, Pd 催化体系有着更高的反应 活性. 正是基于这两类催化体系反应活性的差异, Buchwald 小组 ${ }^{[8]}$ 设计了 $\mathrm{Pd}$ 和 $\mathrm{Cu}$ 两催化体系的序列 $\mathrm{C}-\mathrm{O}$ 偶联反应. 以间溴碘苯为亲电偶联体, 在 $\mathrm{Cu}$ 催化 体系下，首先选择性地实现了芳基碘代物和异丙醇的偶 联; 接着, 在 $\mathrm{Pd}$ 催化体系下实现了烯丙醇和芳基溴代 物的偶联，从而实现了高专一选择性二芳基卤代烃的二 烷氧基化反应(Scheme 8).

\section{3 在天然产物合成中的应用}

Beller 小组 ${ }^{[16]}$ 运用他们发展的催化体系，成功的运 用于麻醉药 butoxycaine 的合成(Scheme 9). 值得一提的 是，在 $\mathrm{Pd}$ 催化的 $\mathrm{C}-\mathrm{O}$ 偶联反应中, $1 \mathrm{~mol} \%$ 的催化剂用 


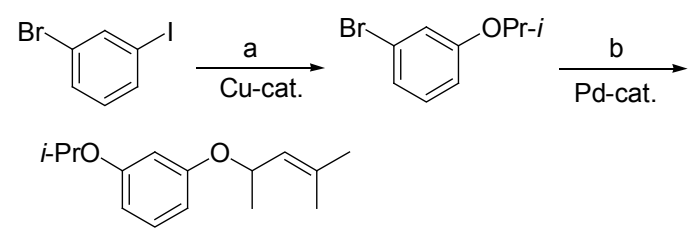

Reagents and conditions: (a) $10 \mathrm{~mol} \% \mathrm{Cul}, 20 \mathrm{~mol} \%$ 1,10-phenanthroline, 2 equiv. $\mathrm{Cs}_{2} \mathrm{CO}_{3}$, i-PrOH neat, $110{ }^{\circ} \mathrm{C}, 24 \mathrm{~h}, 81 \%$; (b) 3 $\mathrm{mol} \% \mathrm{Pd}(\mathrm{OAc})_{2}, 3.6 \mathrm{~mol} \%$ of $\mathbf{L 9}, 1.5$ equiv. $\mathrm{Cs}_{2} \mathrm{CO}_{3}, \mathrm{Bu}_{3} \mathrm{~N}, 50{ }^{\circ} \mathrm{C}$, $18 \mathrm{~h}, 84 \%$

\section{Scheme 8}

量即可; 而且反应结束后, 无需纯化便可投入下一步反 应，以两步 74\%的收率得到 4-丁氧基苯甲酸.
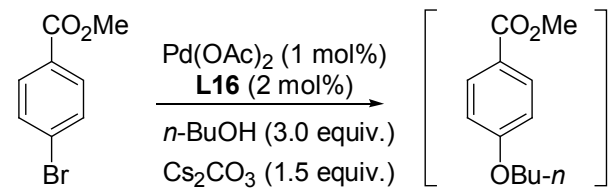

toluene, $80^{\circ} \mathrm{C}, 3 \mathrm{~h}$
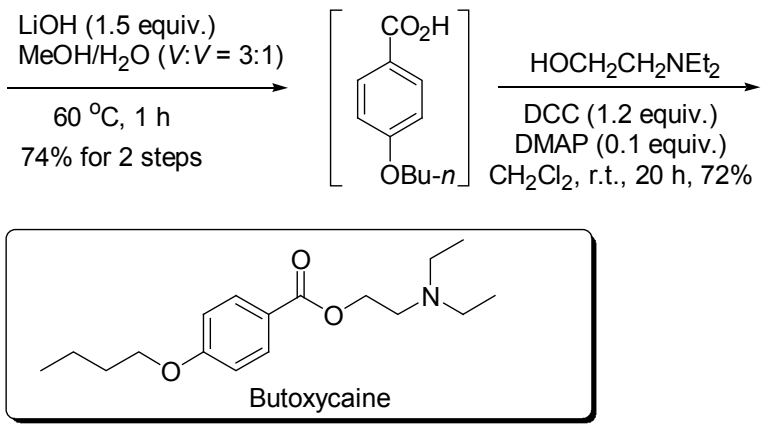

Scheme 9

Buchwald 小组 ${ }^{[7]}$ 在合成抑郁药 MKC-242 时, 以芝 麻酚为起始原料, $\mathrm{Pd}$ 催化的分子内 $\mathrm{C}-\mathrm{O}$ 偶联为关键反 应步骤, 从而可以高效合成 1,4-苯并二啞烷中间体, 接 着通过两步常规反应得到目标化合物(Scheme 10).<smiles>CC(F)(F)N(CCCOc1ccc2c(c1)OCO2)C[C@@H](O)COc1ccc(C=C[C@H]2Oc3ccc(O)cc3O2)cc1Br</smiles>

Sesamol $\mathrm{Pd}(\mathrm{OAc})_{2}(3 \mathrm{~mol} \%)$ L5 (3.5 mol\%)

$\mathrm{K}_{3} \mathrm{PO}_{4}$ (1.5 equiv.) toluene, $70^{\circ} \mathrm{C}, 90 \mathrm{~h}$
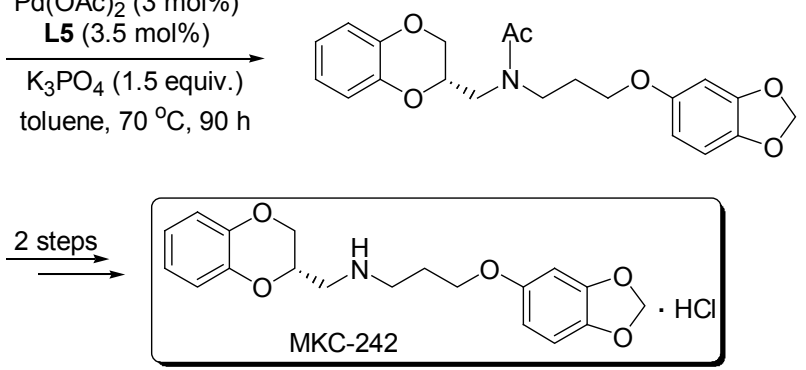

Scheme 10

\section{4 结语与展望}

在过去的 10 多年时间里, 由于新型配体的不断涌 现, $\mathrm{Pd}$ 催化的 $\mathrm{C}-\mathrm{O}$ 反应取得了很多突破, 具体体现在 反应效率、底物的普适性、官能团的兼容性等方面. 但 与相对成熟的 $\mathrm{C}-\mathrm{C}, \mathrm{C}-\mathrm{N}$ 等偶联反应相比, 在高效配 体的设计与合成、反应机理的理解上, $\mathrm{C}-\mathrm{O}$ 偶联反应还 有很多研究工作需要开展. 接下来的 $\mathrm{C}-\mathrm{O}$ 偶联反应研 究工作有可能围绕以下几个方面进行: (1)新配体的设计 与合成. 主要解决富电子大位阻的芳基氯代烃和大位阻 叔醇的偶联; 设计可回收型催化剂, 提高反应经济性; 易合成且稳定的无磷型配体的应用，使反应更为绿色与 环保. (2)加深对 $\mathrm{C}-\mathrm{O}$ 偶联反应机理的理解. 机理的正 确理解对于发展专属的 $\mathrm{C}-\mathrm{O}$ 偶联反应配体和设计与其 它反应的串联反应都将提供更多的理论和实验上的支 持. (3)新型反应媒介的使用. 例如微波取代传统加热、 离子液体取代传统反应媒介对提高反应的经济性和环 保性都将是有益的探索. 随着 $\mathrm{C}-\mathrm{O}$ 偶联反应研究领域 不断的拓展和深入，新颖的高效配体和反应将不断涌 现，偶联反应将逐渐成为十分重要且有效的构筑 $\mathrm{C}-\mathrm{O}$ 键的合成策略与方法.

\section{References}

[1] Enthaler, S.; Company, A. Chem. Soc. Rev. 2011, 40, 4912.

[2] (a) Fuhrmann, E.; Talbiersky, J. Org. Process Res. Dev. 2005, 9, 206.

(b) Lindley, J. Tetrahedron 1984, 40, 1433.

(c) Quach, T. D.; Batey, R. A. Org. Lett. 2003, 5, 1381.

(d) Brandt, S. V.; Rombouts, F. J. R.; Martinez-Lamenca, C.; Leenaers, J.; Rauws, T. R. M.; Trabanco, A. A. Eur. J. Org. Chem. 2012, 7048.

(e) Swamy, K. C. K.; Kumar, N. N. B.; Balaraman, E.; Kumar, K. V. P. P. Chem. Rev. 2009, 109, 2551.

(f) Sach, N. W.; Richter, D. T.; Cripps, S.; Tran-Dubé, M.; Zhu, H.; Huang, B.; Cui, J.; Sutton, S. C. Org. Lett. 2012, 14, 3886.

(g) Bhadra, S.; Dzik, W. I.; Goossen, L. J. J. Am. Chem. Soc. 2012, 134, 9938 .

(h) Barluenga, J.; Tomá-Gamasa, M.; Aznar, F.; Valdés, C. Angew. Chem., Int. Ed. 2010, 49, 4993.

(i) Simon, M.-O.; Girard, S. A.; Li, C.-J. Angew. Chem., Int. Ed. 2012, 51, 7537.

[3] (a) Jiang, T.-S.; Wang, G.-W. J. Org. Chem. 2012, 77, 9504.

(b) Li, W.; Sun, P. J. Org. Chem. 2012, 77, 8362.

(c) Wang, G.-W.; Yuan, T.-T. J. Org. Chem. 2010, 75, 476.

(d) Wang, X.; Lu, Y.; Dai, H.-X.; Yu, J.-Q. J. Am. Chem. Soc. 2010, 132, 12203.

(e) Desai, L. V.; Malik, H. A.; Sanford, M. S. Org. Lett. 2006, 8, 1141 .

(f) Dick, A. R.; Hull, K. L.; Sanford, M. S. J. Am. Chem. Soc. 2004, 126, 2300.

(g) Dick, A. R.; Hull, K. L.; Sanford, M. S. J. Am. Chem. Soc. 2004, 126, 9542 .

(h) Desai, L. V.; Stowers, K. J.; Sanford, M. S. J. Am. Chem. Soc. 2008, 130, 13285. 
(i) Neufeldt, S. R.; Sanford, M. S. Org. Lett. 2010, 12, 532.

(j) Ren, Z.; Mo, F.; Dong, G. J. Am. Chem. Soc. 2012, 134, 16991.

[4] (a) Wolter, M.; Nordmann, G. E.; Buchwald, S. L. Org. Lett. 2002, 4, 973.

(b) Zhang, H.; Ma, D.; Cao, W. Synlett 2007, 243.

[5] (a) Palucki, M.; Wolfe, J. P.; Buchwald, S. L. J. Am. Chem. Soc. 1996, $118,10333$.

(b) Palucki, M.; Wolfe, J. P.; Buchwald, S. L. J. Am. Chem. Soc. 1997, 119, 3395.

(c) Mann, G.; Hartwig, J. F. J. Org. Chem. 1997, 62, 5413.

(d) Mann, G.; Hartwig, J. F. Tetrahedron Lett. 1997, $38,8005$.

(e) Parrish, C. A.; Buchwald, S. L. J. Org. Chem. 2001, 66, 2498.

[6] Torraca, K. E.; Kuwabe, S.-I.; Buchwald, S. L. J. Am. Chem. Soc. 2000, 122, 12907.

[7] Kuwabe, S.-I.; Torraca, K. E.; Buchwald, S. L. J. Am. Chem. Soc. 2001, 123, 12202.

[8] Vorogushin, A. V.; Huang, X.; Buchwald, S. L. J. Am. Chem. Soc. 2005, 127,8146

[9] Watson, D. A.; Su, M.; Teverovskiy, G.; Zhang, Y.; GarciáFortanet, J.; Kinzel, T.; Buchwald, S. L. Science 2009, 325, 1661.

[10] Wu, X.; Fors, B. P.; Buchwald, S. L. Angew. Chem., Int. Ed. 2011, $50,9943$.

[11] (a) Zhu, R.; Buchwald, S. L. Angew. Chem., Int. Ed. 2012, 51, 1926.

(b) Dash, P.; Janni, M.; Peruncheralathan, S. Eur. J. Org. Chem. 2012, 4914.

[12] Mann, G.; Incarvito, C.; Rheingold, A. L.; Hartwig, J. F. J. Am. Chem. Soc. 1999, 121, 3224.

[13] (a) Shelby, Q.; Kataoka, N.; Mann, G.; Hartwig, J. F. J. Am. Chem. Soc. 2000, 122, 10718.

(b) Kataoka, N.; Shelby, Q.; Stambuli, J. P.; Hartwig, J. F. J. Org. Chem. 2002, 67, 5553.
[14] Singer, R. A.; Doré, M.; Sieser, J. E.; Berliner, M. A. Tetrahedron Lett. 2006, 47, 3727.

[15] Withbroe, G. J.; Singer, R. A.; Sieser, J. E. Org. Process Res. Dev. 2008, 12, 480 .

[16] Gowrisankar, S.; Sergeev, A. G.; Anbarasan, P.; Spannenberg, A.; Neumann, H.; Beller, M. J. Am. Chem. Soc. 2010, 132, 11592.

[17] Gowrisankar, S.; Neumann, H.; Beller, M. ChemCatChem 2011, 3, 1439.

[18] Gowrisankar, S.; Neumann, H.; Beller, M. Chem. Eur. J. 2012, 18, 2498.

[19] Maligres, P. E.; Li, J.; Krska, S. W.; Schreier, J. D.; Raheem, I. T. Angew. Chem., Int. Ed. 2012, 51, 9071.

[20] (a) Watanabe, M.; Nishivama, M.; Koie, Y. Tetrahedron Lett. 1999, $40,8837$.

(b) Ylijoki, K. E. O.; Kündig, E. P. Chem. Commun. 2011, 47, 10608 .

[21] Jing, X. B.; Yan, C. G.; Sun, J.; Wang, L.; An, L. Chin. Chem. Lett. 2004, 15, 1392.

[22] (a) Neogi, A.; Majhi, T. P.; Achari, B.; Chattopadhyay, P. Eur. J. Org. Chem. 2008, 330.

(b) Bhattacharya, D.; Behera, A.; Hota, S. K.; Chattopadhyay, P. Synthesis 2011, 585.

[23] Meng, T.; Zhang, W.-X.; Zhang, H.-J.; Liang, Y.; Xi, Z. Synthesis 2012, 2754.

[24] Mann, G.; Hartwig, J. F. J. Am. Chem. Soc. 1996, 118, 13109.

[25] (a) Widenhoefer, R. A.; Zhong, H. A.; Buchwald, S. L. J. Am. Chem. Soc. 1997, 119, 6787.

(b) Widenhoefer, R. A.; Buchwald, S. L. J. Am. Chem. Soc. 1998, 120,6504

(c) Aranyos, A.; Old, D. W.; Kiyomori, A.; Wolfe, J. P.; Sadighi, J. P.; Buchwald, S. L. J. Am. Chem. Soc. 1999, 121, 4369. 